\title{
Noise in distributed erbium-doped fibers
}

Rottwitt, Karsten; Povlsen, Jørn Hedegaard; Bjarklev, Anders Overgaard; Lumholt, Ole; Pedersen, Bo; Rasmussen, Thomas

Published in:

I E E E Photonics Technology Letters

Link to article, DOI:

$10.1109 / 68.196010$

Publication date:

1993

Document Version

Publisher's PDF, also known as Version of record

Link back to DTU Orbit

Citation (APA):

Rottwitt, K., Povlsen, J. H., Bjarklev, A. O., Lumholt, O., Pedersen, B., \& Rasmussen, T. (1993). Noise in distributed erbium-doped fibers. I E E E Photonics Technology Letters, 5(2), 218-221.

https://doi.org/10.1109/68.196010

\section{General rights}

Copyright and moral rights for the publications made accessible in the public portal are retained by the authors and/or other copyright owners and it is a condition of accessing publications that users recognise and abide by the legal requirements associated with these rights.

- Users may download and print one copy of any publication from the public portal for the purpose of private study or research.

- You may not further distribute the material or use it for any profit-making activity or commercial gain

- You may freely distribute the URL identifying the publication in the public portal

If you believe that this document breaches copyright please contact us providing details, and we will remove access to the work immediately and investigate your claim 


\title{
Noise in Distributed Erbium-Doped Fibers
}

\author{
Karsten Rottwitt, Jørn H. Povlsen, Anders Bjarklev, Ole Lumholt, \\ Bo Pedersen, and Thomas Rasmussen
}

\begin{abstract}
Theoretical limits in noise figure for a long-haul transmission line based on lumped amplification are contrasted with distributed amplification. The latter results in a reduction of approximately $60 \%$ of the required number of pump power stations. The distributed optical amplification is provided by an erbium-doped fiber and comparisons of aluminum and germanium as codopant materials are shown. The pump power consumption and noise figure are analyzed with respect to the background loss.
\end{abstract}

\section{INTRODUCTION}

$I^{N}$ $\mathrm{N}$ recent years there has been a large research effort in the field of optical amplification in long haul transmission links. The majority of this work has been done on system configurations which are based on the use of lumped erbium doped fiber amplifiers (EDFA's). However, lately another approach has been suggested [1] which makes use of distributed erbium-doped fibers (d-EDF's), in which the whole transmission fiber is erbium-doped, this provides loss compensation over the total fiber length. Common for the systems with either lumped or distributed erbium doped fibers are that they apply optical amplification which is accompanied by the addition of amplified spontaneous emission (ASE). A tradeoff exists between pump power consumption, noise figure and spacing between pump power stations, and all these properties have to be considered in order to enable a fair comparison between different approaches. In the following we will focus on this tradeoff and theoretical noise figures of the d-EDF are contrasted with noise figures of a transmission link based on ideal lumped amplification over an equal fiber span. Considerations concerning comparisons between noise performance in transmission lines based on lumped- and distributed-amplification have previously been reported [2], [3]. However, we compare two lumped gain configurations with distributed amplification and furthermore, we focus on nonlinear transmission.

\section{NoISE Figure CONSIDERATIONS}

In order to evaluate the performance of a d-EDF it is appropriate to compare distributed amplification with lumped amplification over an equivalent total fiber span. When considering a transmission link based on lumped gain, we will consider amplification achieved with use of EDFA's pumped at $980 \mathrm{~nm}$. The noise figure of each

Manuscript received August 25, 1992; revised November 16, 1992. The authors are with the Center for Broadband Telecommunications, Electromagnetics Institute, Technical University of Denmark, DK-2800 Lyngby, Denmark.

IEEE Log Number 9206419.
EDFA will be considered as ideal. This means that complete inversion is assumed and thus the noise figure $F=2-1 / G$ [4]. This approximation is applicable as we wish to show that distributed amplification is preferable compared to lumped amplification when the design of the d-EDF is optimized. To assure transparency of a lumped gain transmission link, the gain $G$ of each amplifier has to compensate for the attenuation of the passive optical fiber section between two amplifiers, thus $G=\exp (\alpha L)$, where $\alpha$ is the attenuation constant and $L$ the length of the passive optical fiber section. The path average signal power along the transmission link based on lumped gain is $\langle P\rangle=P_{0} \cdot R_{g}$ where $P_{0}$ is the launched signal power and $R_{g}=(G-1) /(G \ln (G))$. In general two configurations of transparent lumped gain links exist [3]. In configuration $A$, Fig. 1(a) denoted as the power amplifier configuration, the signal from the signal source is first amplified and then transmitted through a passive fiber section. Fig. 1(b) shows configuration $B$, denoted the preamplifier configuration, where the signal is directly launched to a passive fiber and then amplified. Each configuration consists of $N$ identical blocks containing an amplifier and a passive fiber section. In configuration $A$ the mean signal power along the transmission link is $\langle P\rangle_{A}=P_{A} \cdot R_{g} G$, where $P_{A}$ is the launched signal power. The number of ASE photons after one block is $n_{\mathrm{ASE}}=(F \cdot G-1) / G$. After $N$ blocks the number of ASE photons is $n_{\mathrm{ASE}}=N \cdot(F \cdot G-1) / G$, equal to a noise figure of $F_{A}=N \cdot(F-1 / G)+1$. In configuration $B$ the mean signal power is $\langle P\rangle_{B}=P_{B} \cdot R_{g}$, where $P_{B}$ is the launched signal power and the number of ASE photons after one block is $n_{\mathrm{ASE}}=(F \cdot G-1)$, resulting in $n_{\mathrm{ASE}}=N \cdot(F \cdot G-1)$ ASE photons after $N$ blocks equivalent to the noise figure $F_{B}=N \cdot(F \cdot G-1)$ +1 .

In long distance transmission the system is considered as nonlinear if the path average power is high enough to support solitary pulses and linear if the path average signal power is sufficiently below the soliton power [5]. Thus, a comparison between various systems may be performed by comparing signal to noise ratios rather than noise figures. Furthermore, a fair comparison is performed on the basis of an equal signal path average power. In general, the signal to noise ratio at the signal output end of the transmission line, $(S / N)_{\text {out }}$, is related to the signal to noise ratio at the signal input end, $(S / N)_{\text {in }}$, through the noise figure of the complete transmission line $F_{i},(S / N)_{\text {out }}=(S / N)_{\text {in }} / F_{i}, i$ equals $A$ or $B$ in the power- or preamplifier-configuration, respectively. Assuming a coherent signal source the $(S / N)_{\text {in }}$ is equal 


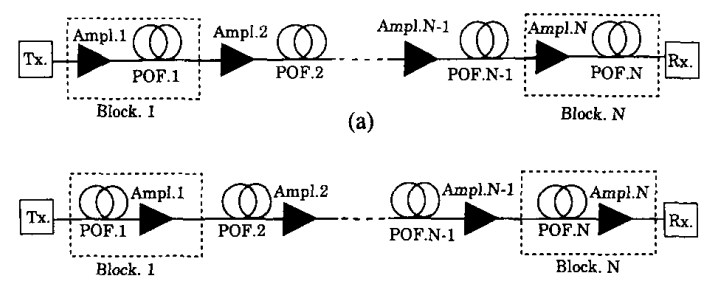

(b)

Fig. 1. (a) the power amplifier configuration. (b) the preamplifier configuration. Ampl. $n$ represents amplifier no. $n$ having gain $G$ and noise figure $F$. POF, $n$ represents the passive fiber section no. $n$. $T x$ is the transmitter and $\mathrm{Rx}$ the receiver.

to the mean number of emitted signal photons, which is proportional to the launched signal power [6]. In configuration $A$ the signal to noise ratio at the signal output end is $P_{A} / F_{A}=\langle P\rangle_{A} /\left(F_{A} \cdot R_{g} G\right)$, whereas the signal to noise ratio at the signal output end in configuration $B$ is $P_{B} / F_{B}$ $=\langle P\rangle_{B} /\left(F_{B} \cdot R_{g}\right)$. In the following, the mean signal power level is fixed and therefore the focus will be on the two effective noise figures $\mathrm{NF}_{A}=F_{A} \cdot R_{g} G$ and $\mathrm{NF}_{B}=F_{B} \cdot R_{g}$, when lumped gain is considered.

For comparison with distributed amplification a long haul transmission line based on lumped gain is subdivided into sections each of $100 \mathrm{~km}$ lengths. One section is then further divided into $N$ parts of length $100 / N \mathrm{~km}$. In Fig. 2 the effective noise figure of the $100 \mathrm{~km}$ fiber span is shown as a function of the number of parts $\mathrm{N}$ equal to the number of amplifiers within the span. From the figure it is seen that two amplifiers give the effective noise figures $\mathrm{NF}_{A}=14.4 \mathrm{~dB}$ and $\mathrm{NF}_{B}=13.5 \mathrm{~dB}$. If $D$ is the total attenuation over $100 \mathrm{~km}$ fiber without any amplification, the asymptotic value of the effective noise figure, equivalent to an infinite number of amplifiers, is $F_{\text {lim }}=2 \ln (D)$ +1 . This value is the noise figure of an ideal transmission line, where the loss is compensated at every point along the fiber. The noise figure of a $d$-EDF has this value as lower limit.

\section{EVALUATION OF THE d-EDF}

We consider two fibers both based on a $\mathrm{GeO}_{2} / \mathrm{SiO}_{2}$ host glass composition. One fiber is purely codoped with germanium, denoted the $\mathrm{Ge} / \mathrm{Er}$ fiber. The other fiber has an additional low aluminum codopant concentration, denoted the $\mathrm{Al} / \mathrm{Ge} / \mathrm{Er}$ fiber, which assures that the cross section spectra are dominated by the aluminum spectra as shown in [7]. The cross section spectra used are similar to those presented in [4]. The analysis is based on an accurate numerical model for d-EDF [4]. Throughout the letter the length of the d-EDF is $100 \mathrm{~km}$ and the fiber design as in [8], with a numerical aperture of 0.2 and an erbium dopant radius 0.8 times the fiber core radius. The $\mathrm{d}$-EDF is bidirectionally pumped at $1.48 \mu \mathrm{m}$ and the pump power values are the total launched pump power. For bidirectionally pumped d-EDF, the signal power excursions are very low typically less than $\pm 2 \mathrm{~dB}$ [9], and the launched signal power equals the path average signal

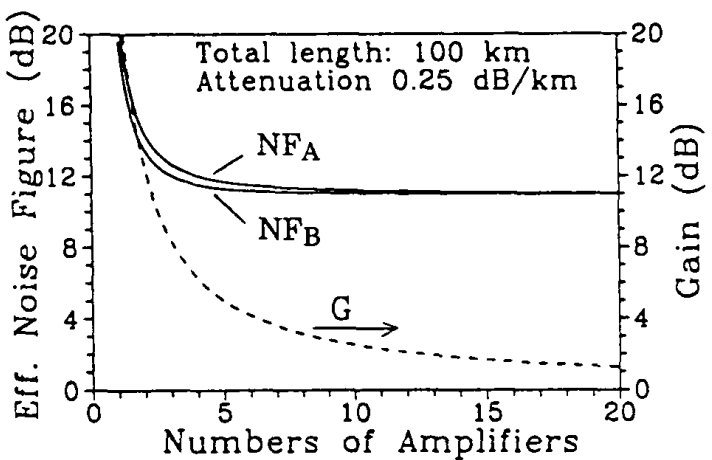

Fig. 2. Effective noise figures $\mathrm{NF}_{A}$ and $\mathrm{NF}_{B}$ for the power and preamplifier configuration respectively, versus the number of amplifiers within a $100 \mathrm{~km}$ fiber span. The dashed curve display the gain $G$ of each amplifier.

power. It is worth noting that using a coherent $1480 \mathrm{~nm}$ pump light, the Raman effect may cause a small extra gain and the necessary gain due to the erbium must therefore be reduced correspondingly. The noise performance of the transmission line will then be improved as the noise figure in a Raman amplifier is lower than in an EDFA [3]. However, we neglect the Raman effect in the following.

Operating at a fixed signal wavelength the necessary pump power for transparency decreases with increasing erbium dopant concentration until the signal absorption introduced by the erbium results in an overall signal attenuation. This defines an optimum erbium concentration where the pump power is minimized. Fig. 3 displays the necessary pump power for transparency and the corresponding noise figure at this erbium concentration. The actual values of pump power and noise figures are found having a path average signal power of $0.1 \mathrm{~mW}$. From the figure we conclude that the minimum pump power for transparency is achieved for the signal wavelengths 1.534 and $1.554 \mu \mathrm{m}$ for the Ge/Er fiber and $1.562 \mu \mathrm{m}$ for the $\mathrm{Al} / \mathrm{Ge} / \mathrm{Er}$ fiber. In Fig. 3 also the noise figure is shown where the pump power exhibit minimum. However, the noise figure increases with increasing erbium concentration. Hence, the minimum noise figure is achieved when the erbium concentration only is sufficient when an infinite pump power is used to obtain transparency. This defines a concentration here denoted as the critical erbium concentration. Thus, for a fixed signal wavelength the erbium concentration has to be in the range between the critical erbium concentration and the optimum erbium concentration. A reasonable analysis of the noise figures is therefore based on a fixed pump power level. Calculation of the noise figure based on an equal pump power level shows that the most efficient signal wavelengths are coinciding with the wavelengths where the minimum in the necessary pump power for transparency occurs [8]. However, the noise figure in the Ge/Er fiber shows an absolute minimum around the $1.554 \mu \mathrm{m}$ signal wavelength. For a pump power of $100 \mathrm{~mW}$ the noise 


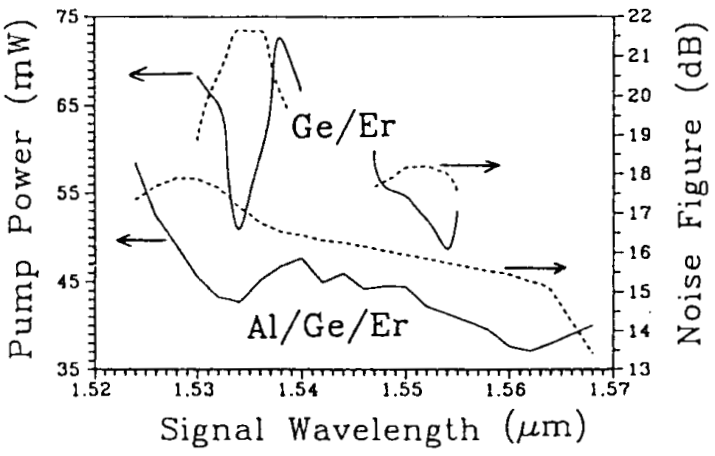

Fig. 3. Minimum pump power for transparency (solid) and the corresponding noise figure (dashed) versus signal wavelength in the $\mathrm{Ge} / \mathrm{Er}$ and the $\mathrm{Al} / \mathrm{Ge} /$ Er fiber.

figure at the signal wavelength $1.534 \mu \mathrm{m}$ is $14.4 \mathrm{~dB}$ where the noise figure at the $1.554 \mu \mathrm{m}$ signal wavelength is 13.1 $\mathrm{dB}$. Therefore, the following analysis is concentrated on the optimum signal wavelengths $1.554 \mu \mathrm{m}$ for the $\mathrm{Ge} / \mathrm{Er}$ fiber and $1.562 \mu \mathrm{m}$ for the $\mathrm{Al} / \mathrm{Ge} / \mathrm{Er}$ fiber. Further calculations have shown that the above concluded optimum signal wavelengths are identical with the signal wavelengths resulting in the lowest signal excursions, and additionally, they are independent on the fiber length and the erbium dopant radius relative to the fiber core radius.

In d-EDF the background loss is very essential due to the relative long length of fiber. For the above conclusions it is assumed that the background loss in both fibers are $0.23 \mathrm{~dB} / \mathrm{km}$. For the d-EDF purely codoped with germanium this is a realistic background loss [10]. However, introducing aluminum may lead to an additional background loss even for a very low aluminum codopant concentration. Furthermore, the background loss of a d-EDF may be increased due to introduced impurities in the fabrication process [1]. In the case of transparency along the whole fiber, an increased background loss causes an increase in both the necessary pump power for transparency and in the generation of ASE [4]. Calculating the necessary pump power for transparency as a function of the background loss, with fixed noise figures, the results displayed in Fig. 4 are achieved. From Fig. 2 it is seen that in order to obtain an effective noise figure better than 13 $\mathrm{dB}$ at least three pump power stations are required within a $100 \mathrm{~km}$ fiber span in a transmission link based on lumped gain. In d-EDF noise figures of less than $13 \mathrm{~dB}$ is very pump power consuming in the $\mathrm{Ge} / \mathrm{Er}$ fiber even for very low background losses according to Fig. 4. At a background loss of $0.23 \mathrm{~dB} / \mathrm{km}$ a total pump power of $110 \mathrm{~mW}$ is necessary. For the $\mathrm{Al} / \mathrm{Ge} / \mathrm{Er}$ fiber, noise figures of $13 \mathrm{~dB}$ or less is realistic for background losses below $0.3 \mathrm{~dB} / \mathrm{km}$. For a background loss of $0.29 \mathrm{~dB} / \mathrm{km}$ the total necessary pump power for transparency is 150 $\mathrm{mW}$. However noise figures of $13 \mathrm{~dB}$ are achievable for realistic pump powers in both fibers. Thus, we conclude that the spacing between two pump power stations may be increased from approximately 30 to $100 \mathrm{~km}$ in a long haul

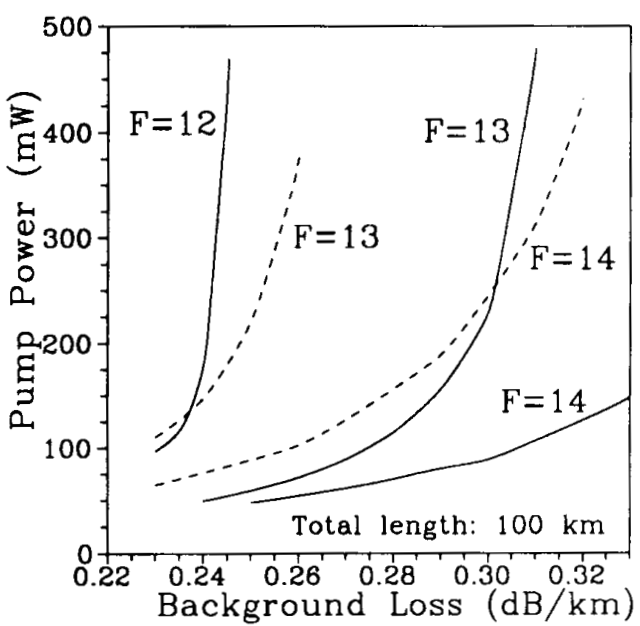

Fig. 4. Necessary pump power for transparency versus background loss at the optimum signal wavelength. The noise figure $F$ is $\mathrm{dB}$ is parameter. The two sets of curves correspond to the $\mathrm{Al} / \mathrm{Ge} / \mathrm{Er}$ fiber (solid) and the $\mathrm{Ge} /$ Er fiber (dashed).

transmission link changing from lumped amplification to distributed amplification. Fig. 4 further illustrates that a noise figure of $13 \mathrm{~dB}$ in both the $\mathrm{Al} / \mathrm{Ge} / \mathrm{Er}$ and the $\mathrm{Ge} /$ Er fiber may be achieved for the same pump power if the background loss of the $\mathrm{Ge} / \mathrm{Er}$ fiber is approximately $0.05 \mathrm{~dB} / \mathrm{km}$ less than the background loss of the $\mathrm{Al} / \mathrm{Ge} / \mathrm{Er}$ fiber.

\section{CONCLUSION}

We have shown that it is possible to reduce the required number of pump power stations by approximately $60 \%$, changing from lumped to distributed amplification in long haul transmission with signal powers large enough to generate solitary pulses. Furthermore, two erbiumdoped distributed fibers are compared. One codoped with germanium and another additionally codoped with a weak aluminum concentration, high enough to ensure aluminum dominated emission and absorption cross section spectra. It is shown that the latter fiber provides the best performance considering both pump power requirements and noise figures, if the background loss does not exceed the background loss in the purely germanium codoped fiber by more than $0.05 \mathrm{~dB} / \mathrm{km}$ for a noise figure of 13 $\mathrm{dB}$.

\section{REFERENCES}

[1] J. R. Simpson, H-T. Shang, L. F. Mollenauer, N. A. Olsson, P. C Becker, K. S. Kranz, P. J. Lemaire and M. J. Neubelt, "Performance of a distributed erbium-doped dispersion-shifted fiber amplifier," J. Lightwave Technol., vol. 9, pp. 228-233, Feb. 1991.

[2] A. Yariv, "Signal-to-noise considerations in fiber links with periodic or distributed optical amplification," Opt. Lett. vol. 15, no. 19, pp. 1064-1066, 1990.

[3] D. N. Chen and E. Desurvire, "Noise performance evaluation of distributed erbium-doped fiber amplifiers with bidirectional pumping at $1.48 \mu \mathrm{m}$," IEEE Photon. Technol. Lett., vol. 4, pp. 52-55, Jan. 1992.

[4] B. Pedersen, A. Bjarklev, J. H. Povlsen, K. Dybdal, and C. C. Larsen, "The design of erbium-doped fiber amplifiers," J.Lightwave Technol., vol. 9, pp. 1105-1112, Sept. 1991. 
[5] L. F. Mollenauer, S. G. Evangiledes and H. A. Haus, "Long-distance soliton propagation using lumped amplifiers and dispersion shifted fiber," J. Lightwave Technol., vol. 9, 1991, pp. 194-197, Feb. 1991.

[6] E. Desurvire, "Spectral noise figure of $\mathrm{Er}^{3+}$-doped fiber amplifiers," IEEE Photon. Technol. Lett., vol. 2, pp. 208-210, Mar. 1990.

[7] S. P. Craig-Ryan, J. F. Massicott, M. Wilson, B. J. Ainslie, and R. Wyatt, "Optical study of low concentration $\mathrm{Er}^{3+}$ fibres for efficient power amplifiers," in Proc. European Conf. Opt. Commun. ECOC'90, vol. 1, pp. 571-574.
[8] K. Rottwitt, J. H. Povlsen, A. Bjarklev, O. Lumholt, B. Pedersen, F. B. Pedersen, and T. Rasmussen, "Detailed analysis of distributed Erbium doped fibre amplifiers," in Proc. Opt. Amplifiers Appl. $O A A^{\prime} 92$, FB6, pp. 178-181.

[9] K. Rottwitt, J. H. Povlsen, A. Bjarklev, O. Lumholt, B. Pedersen, and T. Rasmussen, "Optimum signal wavelength for a distributed erbium-doped fiber amplifier," IEEE Photon. Technol. Lett., vol. 4, pp. 714-717, July 1992.

[10] D. Tanaka, A. Wada, T. Sakai, and R. Yamauchi, "Attenuation free, dispersion shifted fiber doped with distributed erbium," in Proc. Opt. Amplifiers Appl. OAA'90. TuB4, pp. 138-141.

\title{
Enhancement of Optical-Amplifier Noise by Nonlinear Refractive Index and Group-Velocity Dispersion of Optical Fibers
}

\author{
Kazuro Kikuchi
}

\begin{abstract}
The optical-amplifier noise is investigated under the influence of the nonlinear refractive index and the groupvelocity dispersion of optical fibers. By using the newly developed method, we calculate the spectrum of amplified spontaneous emission. The result shows that the positive dispersion is favorable for suppressing the enhancement of the amplifier noise.
\end{abstract}

\section{INTRODUCTION}

$\mathrm{D}$ EVELOPMENT of erbium-doped optical fiber amplifiers enables us to construct ultralong-span optical communication systems by using them as optical repeaters. In such systems, the fiber loss is repeatedly compensated by the optical amplifier gain $G$, which satisfies $G=\exp (\gamma l)$, where $l$ is the repeater separation, and $\gamma$ the loss constant of the fiber.

The amplified spontaneous emission (ASE) from each amplifier is accumulated, and the total ASE power at the output end has been believed to be

$$
P_{\text {ASE }}=h f_{0}\left(1-\frac{1}{G}\right) n_{s p} N \Delta f
$$

where $h f_{0}$ denotes the photon energy, $n_{\text {sp }}$ the spontaneous emission factor, $N$ the number of repeater amplifiers, and $\Delta f$ the bandwidth of the filter in front of the receiver [1]. However, the nonlinear refractive index (Kerr effect) and the group-velocity dispersion of optical fibers may enhance the amplifier noise more than (1).

Manuscript received August 20, 1992; revised September 28, 1992 The author is with the Department of Electronic Engineering, University of Tokyo Tokyo 113, Japan.

IEEE Log Number 9206423.
Gordon and Mollenauer discussed the enhancement of the phase noise due to the nonlinear refractive index [2], and Ryu observed such effect experimentally [3]. On the other hand, Marcuse pointed out that the parametric gain originated from four-wave mixing induces the excess amplifier noise near the zero-dispersion wavelength [4]. Ryu found that the transmitted signal is contaminated by the amplifier noise more seriously in the negative dispersion region than in the positive dispersion region, showing the effect of the four-wave mixing (or the modulational instability) experimentally [5].

In this letter, we analyze the optical amplifier noise under the influence of the nonlinear refractive index and the group-velocity dispersion. Depending on the dispersion and the optical nonlinearity, we classify the operating state of the repeater system into the three categories: I) normal state, II) phase noise state, and III) modulational instability state. By using this classification, the normal ASE power given by (1), the phase noise discussed by Gordon and Mollenauer [2], and the excess noise due to four-wave mixing [4], [5] can be dealt with in a unified manner. From ASE spectra calculated for the three states, we find that the positive dispersion is favorable for suppressing the enhancement of the amplifier noise caused by the modulational instability.

\section{THEORY}

We derive equations which describe the evolution of the amplitude modulation and the phase modulation of the signal light propagating in optical fibers with the nonlinear refractive index and the group-velocity dispersion. The modulation at the frequency of $f_{m}$ is generally 